Acta Crystallographica Section E

Structure Reports

Online

ISSN 1600-5368

\section{Graham Smith, ${ }^{\text {a* Urs D. }}$ Wermuth $^{a}$ and Peter C. Healy ${ }^{b}$}

${ }^{\mathrm{a} S}$ School of Physical and Chemical Sciences, Queensland University of Technology, GPO Box 2434, Brisbane 4001, Australia, and

${ }^{\mathbf{b}}$ School of Science, Griffith University, Nathan 4111, Australia

Correspondence e-mail: g.smith@qut.edu.au

Key indicators

Single-crystal X-ray study

$T=295 \mathrm{~K}$

Mean $\sigma(\mathrm{C}-\mathrm{C})=0.005 \AA$

$R$ factor $=0.046$

$w R$ factor $=0.140$

Data-to-parameter ratio $=8.3$

For details of how these key indicators were automatically derived from the article, see http://journals.iucr.org/e.

\title{
Bis(guanidinium) 5-sulfosalicylate monohydrate
}

The crystal structure of the hydrated proton-transfer compound hydrate of 3-carboxy-4-hydroxybenzenesulfonic acid (5-sulfosalicylic acid) with guanidine, $2 \mathrm{CH}_{6} \mathrm{~N}_{3}{ }^{+}$.$\mathrm{C}_{7} \mathrm{H}_{4} \mathrm{O}_{6} \mathrm{~S}^{2-} \cdot \mathrm{H}_{2} \mathrm{O}$, reveals the presence of an extensively hydrogen-bonded three-dimensional network polymer in which all proton donor and acceptor atoms on the two guanidine cations, the dianionic sulfosalicylate anion and the water molecule are utilized. The structure is significantly different chemically and structurally from that of the previously reported anhydrous guanidinium sulfosalicylate compound $\mathrm{CH}_{6} \mathrm{~N}_{3}^{+} \cdot \mathrm{C}_{7} \mathrm{H}_{5} \mathrm{O}_{6} \mathrm{~S}^{-}$.

\section{Comment}

The acid strength of aromatic sulfonic acids ensures that the formation of proton-transfer compounds will result from their reaction with most Lewis bases. Furthermore, the sulfonate group provides three available $\mathrm{O}$ atoms as proton-accepting centres for hydrogen-bonding associations, enhancing the utility of sulfonates for supramolecular assembly. The structures of the guanidinium salts of a number of sulfonates including aromatic types have been determined because of their potential as optical materials (Russell et al., 1994a,b). This property arises through a combination of the trigonal symmetry of the guanidinium cation with that of the sulfonate group, giving assembly into two-dimensional hydrogenbonded sheet structures which may be extended through interlayer linkages via the third sulfonate $\mathrm{O}$ atom into threedimensional network polymers.

3-Carboxy-4-hydroxybenzenesulfonic acid (5-sulfosalicylic acid, 5-SSA) has the additional interactive carboxylic acid and phenolic functional substituent groups which lend themselves to secondary $n$-dimensional hydrogen-bonding extension. Furthermore, 5-SSA is able to protonate water and several hydrate structures of the acid have been reported: the dihydrate (Attig \& Mootz, 1977; Aliev et al., 1995), the deuterated dihydrate (Attig \& Williams, 1977), the trihydrate (Attig \& Mootz, 1977), and the pentahydrate (Merschenz-Quack \& Mootz, 1990). Many of these have protonated polyaqua species, such as the $\mathrm{H}_{7} \mathrm{O}_{3}{ }^{+}$cation species of the trihydrate (Mootz \& Fayos, 1970). The only reported structures of Lewis base 5-SSA proton-transfer compounds are with aniline (an anhydrate) (Bakasova et al., 1991), theophylline (a monohydrate) (Madarasz et al., 2002), trimethoprim (a dihydrate) (Raj et al., 2003), and guanidine (GU), a 1:1 anhydrate (Zhang et al., 2004). In addition, we have also determined the structures of the proton-transfer compounds of 5-SSA with a set of bicyclic heteroaromatic Lewis bases (Smith et al., 2004). These are with quinoline: quinolinium 5-sulfosalicylate trihydrate; with 8-hydroxyquinoline: 8-hydroxyquinolinium 5-sulfo-
Received 19 March 2004 Accepted 25 March 2004 Online 31 March 2004
(C) 2004 International Union of Crystallography Printed in Great Britain - all rights reserved 
salicylate monohydrate; with 8-aminoquinoline: 8-aminoquinolinium 5-sulfosalicylate dihydrate; and with quinaldic acid, the adduct structure quinolinium-2-carboxylic acid-5sulfosalicylate-quinoline-2-carboxylic acid (1/1). We also prepared a compound with guanidine which is a (2:1) hydrate, reported here: $2(\mathrm{GU})^{+} \cdot(5-\mathrm{SSA})^{2-} \cdot \mathrm{H}_{2} \mathrm{O}$, (I), which is significantly different both chemically and structurally from the Zhang et al. (2004) structure.

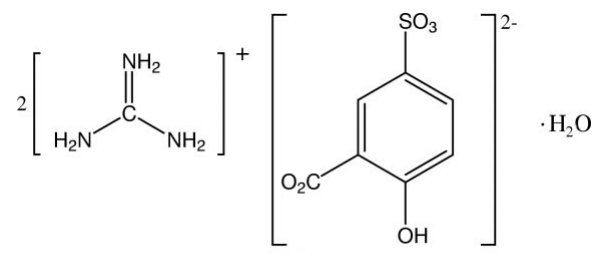

(I)

The structure of (I) shows the presence of a dianionic (5SSA $)^{2-}$ species, arising from the deprotonation of both the sulfonate and the carboxylic acid groups (Fig. 1). As expected with guanidinium salts, there is extensive hydrogen bonding, in this case involving all potential $\mathrm{H}$-atom donors of the $\mathrm{GU}^{+}$ cation in 14 interactions to all sulfonate $\mathrm{O}$ atoms, both carboxylate $\mathrm{O}$ atoms, the phenolic $\mathrm{O}$ atom and the water $\mathrm{O}$ atom acceptor sites of the (5-SSA) ${ }^{-}$anions (Table 1). However, no cyclic $R_{2}^{2}(8) \mathrm{N}-\mathrm{H}$ (guanidine) $\cdots \mathrm{O}$ (sulfonate) interactions are present such as are found in the previously reported $(\mathrm{GU})^{+} \cdot(5-\mathrm{SSA})^{-}$structure (Zhang et al., 2004), where a short $\mathrm{O}-\mathrm{H} \cdots \mathrm{O}$ (carboxyl) linkage [2.611 (2) $\AA$ ] is also found. The result in (I) is a three-dimensional network polymer structure (Fig. 2).

The usual intramolecular Ophenol)...O(carboxyl) hydrogen bond is present $[\mathrm{O} \cdots \mathrm{O}=2.518$ (3) $\AA$ ] in the 5-SSA anion; this distance compares with 2.601 (2) $\AA$ in the $1: 1$ compound (Zhang et al., 2004).

\section{Experimental}

The synthesis of the title compound, (I), was carried out by heating, under reflux, $1 \mathrm{mmol}$ quantities of 3-carboxy-4-hydroxybenzenesulfonic acid (5-sulfosalicylic acid, 5-SSA) and guanidinium carbonate in $50 \mathrm{ml}$ of $50 \%$ ethanol-water for $10 \mathrm{~min}$. After concentration to
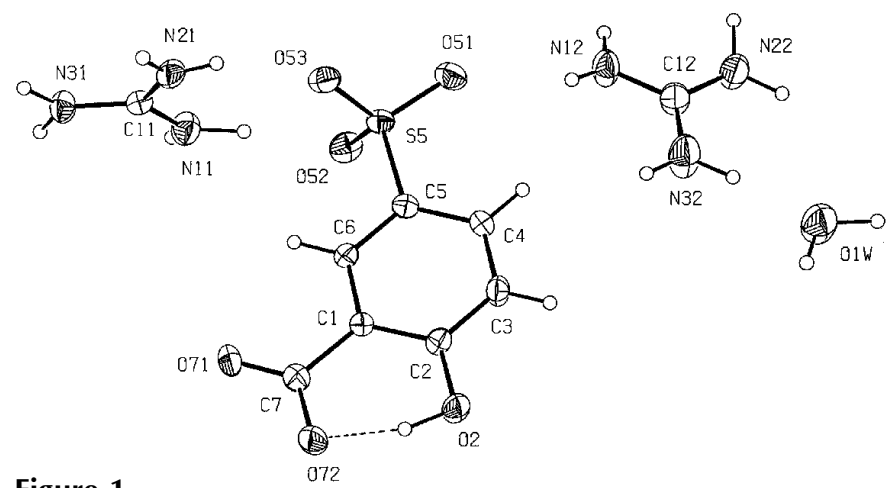

Figure 1

The molecular configuration and atom-naming scheme for (I). Displacement ellipsoids are drawn at the $30 \%$ probability level.

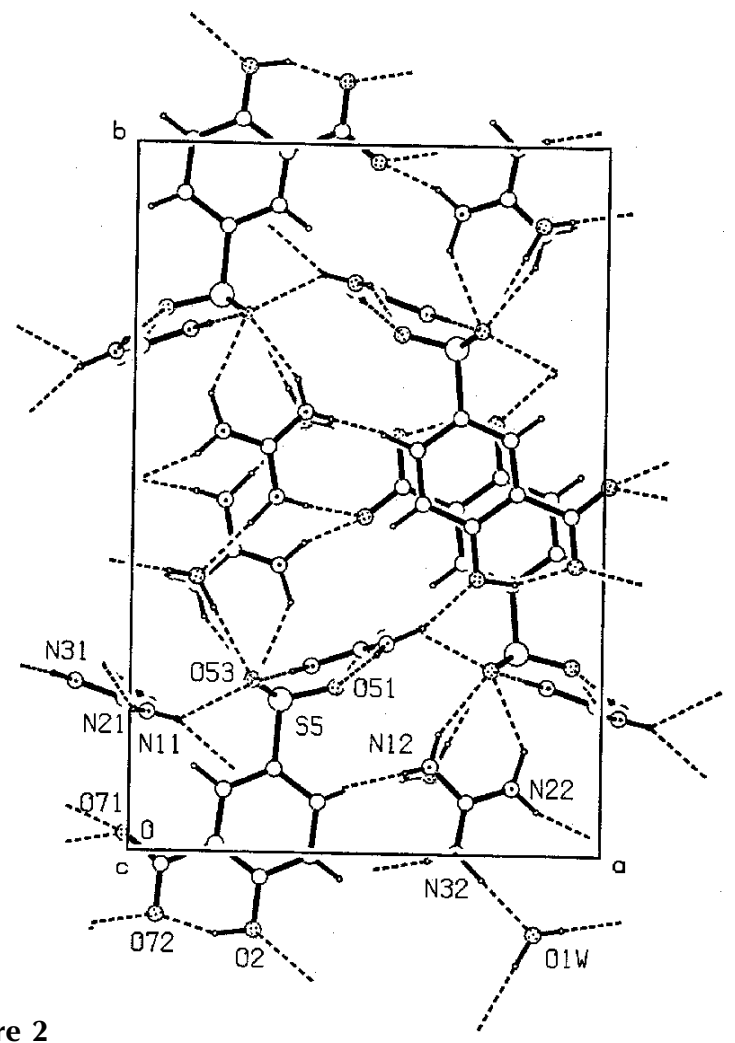

Figure 2

Perspective view of the packing of (I) in the unit cell, viewed down $a$, showing hydrogen-bonding associations as broken lines.

ca $30 \mathrm{ml}$, partial room-temperature evaporation of the hot-filtered solution gave large colourless flat prisms of (I) (m.p. 478.5-484.1 K).

Crystal data

$2 \mathrm{CH}_{6} \mathrm{~N}_{3}{ }^{+} \cdot \mathrm{C}_{7} \mathrm{H}_{4} \mathrm{O}_{6} \mathrm{~S}^{2-} \cdot \mathrm{H}_{2} \mathrm{O}$

$M_{r}=354.35$

Orthorhombic, $P 2_{1} 2_{1} 2_{1}$

$a=12.030(3) \AA$

$b=18.010(5) \AA$

$c=7.290(2) \AA$

$V=1579.5(7) \AA^{3}$

$Z=4$

$D_{x}=1.490 \mathrm{Mg} \mathrm{m}^{-3}$

\section{Data collection}

Rigaku AFC-7 $R$ diffractometer $\omega-2 \theta$ scans

Absorption correction: $\psi$ scan (TEXSAN for Windows:

Molecular Structure

Corporation, 1999)

$T_{\text {min }}=0.895, T_{\max }=0.928$

2428 measured reflections

2173 independent reflections

Refinement

Refinement on $F^{2}$

$R\left[F^{2}>2 \sigma\left(F^{2}\right)\right]=0.046$

$w R\left(F^{2}\right)=0.140$

$S=0.90$

2173 reflections

261 parameters

$\mathrm{H}$ atoms treated by a mixture of independent and constrained refinement
Mo $K \alpha$ radiation

Cell parameters from 25 reflections

$\theta=12.5-17.4^{\circ}$

$\mu=0.25 \mathrm{~mm}^{-1}$

$T=295$ (2) K

Prism, colourless

$0.45 \times 0.35 \times 0.30 \mathrm{~mm}$

1644 reflections with $I>2 \sigma(I)$

$R_{\text {int }}=0.028$

$\theta_{\text {max }}=27.5^{\circ}$

$h=-6 \rightarrow 15$

$k=0 \rightarrow 23$

$l=-3 \rightarrow 9$

3 standard reflections every 150 reflections intensity decay: $0.3 \%$

$w=1 /\left[\sigma^{2}\left(F_{o}^{2}\right)+(0.1 P)^{2}\right.$ $+2.556 P]$

where $P=\left(F_{o}{ }^{2}+2 F_{c}^{2}\right) / 3$

$(\Delta / \sigma)_{\max }=0.015$

$\Delta \rho_{\max }=0.44 \mathrm{e} \AA^{-3}$

$\Delta \rho_{\min }=-0.45{\mathrm{e} \AA^{-3}}^{-3}$

Extinction correction: SHELXL97

Extinction coefficient: 0.059 (4)

Absolute structure: Flack (1983), 79 Friedel pairs

Flack parameter $=0.1(2)$ 
Table 1

Hydrogen-bonding geometry $\left(\AA \mathrm{A}^{\circ}\right)$.

\begin{tabular}{lllll}
\hline$D-\mathrm{H} \cdots A$ & $D-\mathrm{H}$ & $\mathrm{H} \cdots A$ & $D \cdots A$ & $D-\mathrm{H} \cdots A$ \\
\hline $\mathrm{O} 2-\mathrm{H} 2 \cdots \mathrm{O} 72$ & $0.98(6)$ & $1.62(6)$ & $2.518(3)$ & $152(5)$ \\
$\mathrm{O} 1 W-\mathrm{H} 1 A \cdots \mathrm{O} 72^{\mathrm{i}}$ & $0.90(10)$ & $2.11(10)$ & $3.008(7)$ & $179(9)$ \\
$\mathrm{O} 1 W-\mathrm{H} 1 B \cdots \mathrm{O} 53^{\mathrm{ii}}$ & $0.93(11)$ & $2.13(11)$ & $3.055(7)$ & $180(8)$ \\
$\mathrm{N} 11-\mathrm{H} 11 A \cdots \mathrm{O} 53$ & $0.81(7)$ & $2.29(7)$ & $3.018(7)$ & $151(6)$ \\
$\mathrm{N} 11-\mathrm{H} 11 B \cdots \mathrm{O} 51^{\mathrm{iii}}$ & $0.90(5)$ & $2.19(5)$ & $3.089(7)$ & $180(5)$ \\
$\mathrm{N} 12-\mathrm{H} 12 A \cdots \mathrm{O} 72^{\text {iv }}$ & $0.90(4)$ & $2.09(4)$ & $2.987(7)$ & $179(5)$ \\
$\mathrm{N} 12-\mathrm{H} 12 B \cdots \mathrm{O} 52^{\mathrm{v}}$ & $0.89(5)$ & $2.17(5)$ & $3.006(6)$ & $157(4)$ \\
$\mathrm{N} 21-\mathrm{H} 21 A \cdots \mathrm{O} 52^{\text {vi }}$ & $0.83(5)$ & $2.52(5)$ & $3.127(6)$ & $131(5)$ \\
$\mathrm{N} 21-\mathrm{H} 21 A \cdots \mathrm{O} 2^{\text {iv }}$ & $0.83(5)$ & $2.34(5)$ & $3.031(6)$ & $141(5)$ \\
$\mathrm{N} 21-\mathrm{H} 21 B \cdots \mathrm{O} 51^{\text {vii }}$ & $0.89(6)$ & $2.21(6)$ & $3.003(7)$ & $149(5)$ \\
$\mathrm{N} 22-\mathrm{H} 22 A \cdots \mathrm{O} 71^{\mathrm{i}}$ & $0.90(7)$ & $1.94(7)$ & $2.801(6)$ & $159(6)$ \\
$\mathrm{N} 22-\mathrm{H} 22 B \cdots \mathrm{O} 52^{\mathrm{v}}$ & $1.00(9)$ & $2.22(9)$ & $3.043(7)$ & $139(7)$ \\
$\mathrm{N} 31-\mathrm{H} 31 A \cdots \mathrm{O} 53^{\text {vii }}$ & $0.99(7)$ & $1.87(6)$ & $2.860(6)$ & $174(6)$ \\
$\mathrm{N} 31-\mathrm{H} 31 B \cdots \mathrm{O} 52^{\mathrm{iii}}$ & $0.96(7)$ & $1.91(7)$ & $2.873(7)$ & $179(8)$ \\
$\mathrm{N} 32-\mathrm{H} 32 A \cdots \mathrm{O} 1 W$ & $0.94(9)$ & $1.93(9)$ & $2.871(8)$ & $180(8)$ \\
$\mathrm{N} 32-\mathrm{H} 32 B \cdots \mathrm{O} 71^{\text {iv }}$ & $0.81(7)$ & $2.06(8)$ & $2.826(8)$ & $157(8)$ \\
$\mathrm{C} 4-\mathrm{H} 4 \cdots \mathrm{O} 51$ & 0.95 & 2.59 & $2.953(5)$ & 103 \\
\hline
\end{tabular}

Symmetry codes: (i) $1+x, y, z$; (ii) $1-x, y-\frac{1}{2}, \frac{3}{2}-z$; (iii) $x-\frac{1}{2}, \frac{1}{2}-y, 1-z$; (iv)

$\frac{1}{2}-x,-y, \frac{1}{2}+z$; (v) $\frac{1}{2}+x, \frac{1}{2}-y, 1-z$; (vi) $x, y, 1+z$; (vii) $x-\frac{1}{2}, \frac{1}{2}-y, 2-z$.

All $\mathrm{H}$ atoms involved in hydrogen-bonding interactions (those on the guanidinium cation and on the water molecule) were located by difference methods and their positional and isotropic displacement parameters were refined. Others were included in the refinement at calculated positions $(\mathrm{C}-\mathrm{H}=0.95 \AA)$ as riding atoms, with $U_{\text {iso }}(\mathrm{H})=$ $1.2 U_{\text {eq }}(\mathrm{C})$.

Data collection: MSC/AFC Diffractometer Control Software (Molecular Structure Corporation, 1999); cell refinement: $M S C / A F C$ Diffractometer Control Software; data reduction: TEXSAN for Windows (Molecular Structure Corporation, 1999); program(s) used to solve structure: SHELXS97 (Sheldrick, 1997); program(s) used to refine structure: SHELXL97 (Sheldrick, 1997); molecular graphics: PLATON for Windows (Spek, 1999); software used to prepare material for publication: PLATON for Windows.

The authors acknowledge financial support from the School of Physical and Chemical Sciences (Queensland University of Technology) and Griffith University.

\section{References}

Aliev, Z. G., Atovmyan, L. O., Baranova, T. A. \& Ukshe, A. E. (1995). Zh Strukt. Khim. 36, 947-952.

Attig, R. \& Mootz, D. (1977). Acta Cryst. B33, 2422-2427.

Attig, R. \& Williams, J. M. (1977). J. Chem. Phys. 66, 1389-1395.

Bakasova, Z. B., Abdybaliev, D. A., Sharipov, Kh. T., Akbaev, A. A., Ibragimov, B. T., Talipov, S. A. \& Ismankulov, A. I. (1991). Uzbekskii Khim. Zh. pp. 22-25.

Flack, H. D. (1983). Acta Cryst. A39, 876-881.

Madarasz, J., Bombicz, P., Jarmi, K., Ban, M., Pokol, G. \& Gal, S. (2002). J. Therm. Anal. Calorim. 69, 281-290.

Merschenz-Quack, A. \& Mootz, D. (1990). Acta Cryst. C46, 1478-1481.

Molecular Structure Corporation (1999). MSC/AFC Diffractometer Control Software and TEXSAN for Windows (Version 1.06). MSC, 9009 New Trails Drive, The Woodlands, TX 77381, USA.

Mootz, D. \& Fayos, J. (1970). Acta Cryst. B26, 2046-2054.

Raj, S. B., Sethuraman, V., Francis, S., Hemamalini, M., Muthiak, P. T., Bocelli, G., Cantoni, A., Rychlewska, U. \& Warzajtis, B. (2003). CrystEngComm, 5, $70-76$.

Russell, V. A., Etter, M. C. \& Ward, M. D. (1994a). J. Am. Chem. Soc. 116, 1941-1952.

Russell, V. A., Etter, M. C. \& Ward, M. D. (1994b). Chem. Mater. 6, 1206-1217. Sheldrick, G. M. (1997). SHELXS97 and SHELXL97. University of Göttingen, Germany.

Smith, G., Wermuth, U. D. \& White, J. M. (2004). Unpublished results.

Spek, A. L. (1999). PLATON for Windows. September 1999 Version. University of Utrecht, The Netherlands.

Zhang, X.-L., Chen, X.-M. \& Ng, S. W. (2004). Acta Cryst. E60, o453-o454. 